\title{
Development and Application of a Virtual Laboratory for Synthesizing and Analyzing Nanogold Particles
}

\author{
Wernhuar Tarng ${ }^{1 *}$, Chia-Chun Hsie ${ }^{1}$, Chih-Ming Lin ${ }^{2}$, Chi-Young Lee ${ }^{3}$ \\ ${ }^{1}$ Graduate Institute of Human Resource and e-Learning Technology, National Hsinchu University of \\ Education, 521 Nanda Road, Hsinchu, Taiwan. \\ ${ }^{2}$ Department of Applied Science, National Hsinchu University of Education, 521 Nanda Road, Hsinchu, \\ Taiwan. \\ ${ }^{3}$ Department of Materials Science and Engineering, National Tsing Hua University, No. 101, Section 2, \\ Kuang-Fu Road, Hsinchu, Taiwan.
}

* Corresponding author: Tel.: +886-3-5213132\#5903; email: wtarng@nhcue.edu.tw Manuscript submitted November 17, 2015; accepted March 28, 2016.

doi: $10.17706 /$ jcp.12.3.270-283

\begin{abstract}
In this study, a virtual laboratory for synthesizing and analyzing nanogold particles is developed by using the virtual reality technology and situated learning theory. The users can conduct a virtual experiment to become familiar with the process of synthesizing nanogold and understand that nanogold particles in different sizes can be produced by mixing chloroauric acid $\left(\mathrm{HAuCl}_{4}\right)$ with sodium citrate $\left(\mathrm{Na}_{3} \mathrm{C}_{6} \mathrm{H}_{5} \mathrm{O}_{7}\right)$ in different concentrations. A further analysis can be performed by observing the 3D structure of nanogold using the virtual transmission electron microscope to investigate its property within the nanoscale spatial domain. The virtual laboratory developed in this study has been applied in university chemistry courses and the learning objective is to increase students' knowledge in nanotechnology through the operation of virtual experiments. A teaching experiment has been conducted to investigate whether the virtual experiment can enhance their learning achievements, and the results show that it is more effective than a real experiment. Questionnaire results also reveal that most students held positive attitudes towards the virtual laboratory, and they thought it could enhance their interest and motivation in learning nanotechnology.
\end{abstract}

Key words: Virtual reality, situated learning, virtual laboratory, nanotechnology.

\section{Introduction}

Nanotechnology is an emerging technology in recent years. Richard Feynman [1], an American theoretical physicist and Nobel Prize laureate, proposed in 1959 at the California Institute of Technology "There is Plenty of Room at the Bottom," which became one of the 20th Century science's classic lectures. Feynman talked about nanotechnology before the word existed, and he said "All of the information which all of mankind has ever recorded in books can be carried in a pamphlet in your hand." In spite of the advancement in relevant research and technologies, nanotechnology hasn't become a well-known scientific discipline until the last two decades. Nowadays, the advanced countries around the world have dedicated to the development of nanotechnology, which is considered as the new hope of economy in the 21th Century. Nanotechnology has brought a great change to our daily lives, so scientists predict that it will lead to another industrial revolution.

Since nanotechnology has a great impact on national competitiveness, many countries have spent a lot of 
money and manpower to set up its development environment. In 2003, the Ministry of Science and Technology (MOST), Taiwan started the National Nanoscience and Nanotechnology Program (NNNP), which was aimed at establishing a nanotechnology personnel training mechanism for the promotion and development of the local nanotechnology industry in Taiwan [2]. To further extend the training mechanism to support the education of students in high schools and universities, the Ministry of Education (MOE), Taiwan launched the National Nanoscience Training Project (NNTP) to reinforce nanotechnology education in different grades and enhance nanotechnology literacy of the general public [3].

The definition of nanotechnology is manipulation of matter on an atomic, molecular, and supramolecular scale [4]. A more generalized description is established by the U.S. National Nanotechnology Initiative, which defines nanotechnology as the manipulation of matter with at least one dimension sized from 1 to $100 \mathrm{~nm}$ [5]. When the size is reduced to nanoscale, the property of a material, such as its transmittance, electrical conductivity and magnetism, may change greatly. In other words, the material will become a new substance under nanoscale. Taking nanogold as an example, it is different from gold in terms of melting points and hardness. Due to the effect of surface plasmon resonance, the absorption peak of nanogold varies as the wavelength of light reaches $520 \mathrm{~nm}$, thus changing its color from gold to red. Recently, nanogold is widely applied in electronics, optics, and biology, especially in biochips and the localized surface plasmon resonance (LSPR) sensors [6].

The smallest objects that can be seen with the naked eye are about $0.1 \mathrm{~mm}$. To observe smaller objects such as biological cells, scientists invented the optical microscope, giving the human a better perception of the natural biological world. The optical microscope can only be used to view objects larger than the wavelength of visible light. To observe matter approaching the atomic size, the wavelength of light has to be shorter than that of visible light. To enable such observation, an energy beam has been used for diffraction imaging between atoms. Recently, many high resolution microscopes such as the atomic force microscope (AFM) [7], transmission electron microscope (TEM) [8] and magnetic force microscope (MFM) [9] have been invented to observe the nanostructures of different materials.

Nanogold particles are one of the materials frequently mentioned in nanomedical research regarding their conjugation to specific drug to enable its delivery to target cells [10]. Several approaches have been applied to produce nanogold, and the method most commonly used was proposed by Turkevitch et al. [11]. By mixing chloroauric acid $\left(\mathrm{HAuCl}_{4}\right)$ with citric acid $\left(\mathrm{Na}_{3} \mathrm{C}_{6} \mathrm{H}_{5} \mathrm{O}_{7}\right)$ solution, the nanogold particles of $20 \mathrm{~nm}$ size can be produced. Frens [12] synthesized nanogold particles of varying sizes by changing the concentration of sodium citrate when mixing with chloroauric acid. After forming the nanogold particles, the unreacted citric acid ions and other negative charged particles will attach to the surface of nanogold particles, causing them to carry negative charges. The mutual repulsive effect exists among these particles since each particle carries the same type of electrical charge. Thus, nanogold particles can stably suspend in the solution without aggregation or precipitation, so the solution acquired is practically a colloidal solution.

Synthesizing and analyzing nanogold particles requires instruments for conducting the chemical experiment and observing their nanostructure. Besides, students must be aware of the correct procedure in operating the instruments, and learn to properly dispose of harmful wastes produced by the experiment. Since nanostructures cannot be seen with the naked eye or by general microscopes, an electron microscope or special equipment has to be used for observation. Recently, many high resolution microscopes have been invented for observing nanomaterials. However, they are very expensive and vulnerable to damage due to improper operation and thus most schools can't provide students with such equipment. As a result, students can only verify their experimental results according to the data on textbooks or images provided by their teachers.

Virtual reality (VR) is a human-computer interface to create a 3D virtual world for simulating the real 
environments through our sense organs [13]. Based on the sensor data such as the current orientation and position, a VR system can detect the user's reaction to provide interactive feedback in real time for simulating his or her visual and audio perception. VR is composed of three elements, that is, interaction, immersion, and imagination, called the 3I of VR [14]. It is often applied to create a virtual learning environment not easily seen in the real world. A virtual and realistic environment can avoid the danger and reduce the cost in the real world while satisfying the major requirements [15]. Its educational applications include the simulation of expensive instrument in a laboratory for students to conduct virtual experiments and field surveys in places unreachable by them. Furthermore, VR provides a safe learning environment where students can obtain knowledge and learning experience from trial and error.

VR can simulate realistic situations by 3D animation, so it is useful for presenting abstract concepts, theories and experimental processes to enhance immersion in learning activities. Some researchers considered VR effective in science education according to the results of case studies. For example, Visher [16] is a teaching module using the VR technology to simulate chemical reactions. Experimental results revealed that students could develop an intuition to help learning in a positive way when exploring the relationship between various variables in a chemical reaction. Also, there was a positive feedback from students since it could help them understand scientific concepts. Tarng et al. [17] developed a VR teaching module to simulate the phenomenon of butterfly effects, which can be used to observe the nanostructure of photonic crystals on butterfly wings. The experimental results indicated that it is more effective than traditional teaching materials in improving the learning achievements. In addition, it could also increase students' learning motivation and interest.

Situated learning was proposed by Brown et al. [18], and the purpose is for students to learn under realistic or simulated situations. Situated learning allows learners to participate in learning activities with repeated reflection and feedback so as to increase learning effectiveness. Aside from constructing meaningful scientific knowledge, learners can also apply their knowledge to solve practical problems. It is similar to the ideas of "Learning by Doing" [19] and "Learning from Experiences" [20], and the goal is to carry out conceptual learning according to practical demand in the real life. The emergence of situated learning have attracted a number of relevant theories and research, such as constructivism, anchored instruction and cognitive apprenticeship, and these theories have been applied in various teaching models [21].

The objective of using learning strategy in situated learning is to help students observe, participate, and discover to develop their critical thinking and problem solving skills. Collins [22] proposed the combination of interweaving, connecting, reflecting, cycling and using multimedia for the design of teaching activities to place learners in an abundant and realistic learning situation to construct their learning process and knowledge structure automatically. As a result, it is emphasized that the learning situations must be suitable for learners such that they can make internal interpretation about the learning experience and obtained knowledge for applications in the future so as to enhance their problem solving ability.

In this study, a virtual laboratory for synthesizing and analyzing nanogold particles is developed based on the VR technology and the situated learning theory. The users can conduct a virtual experiment to become familiar with the process of synthesizing nanogold and understand that the nanogold particles of different sizes can be produced by mixing chloroauric acid with sodium citrate in different concentrations. The users can also observe the structures of nanogold particles by using the virtual transmission electron microscope to analyze their properties. A teaching experiment has been performed to investigate if the virtual experiment can enhance the learning achievement of students. Also, a questionnaire survey has been conducted to understand their attitudes after using the virtual laboratory. The rest of this paper is organized as follows. Section 2 demonstrates the system architecture and operational procedure of the 
virtual experiment. Section 3 describes the teaching experiment and its analytical results. Section 4 is the conclusion.

\section{System Design}

\subsection{Operational Procedure}

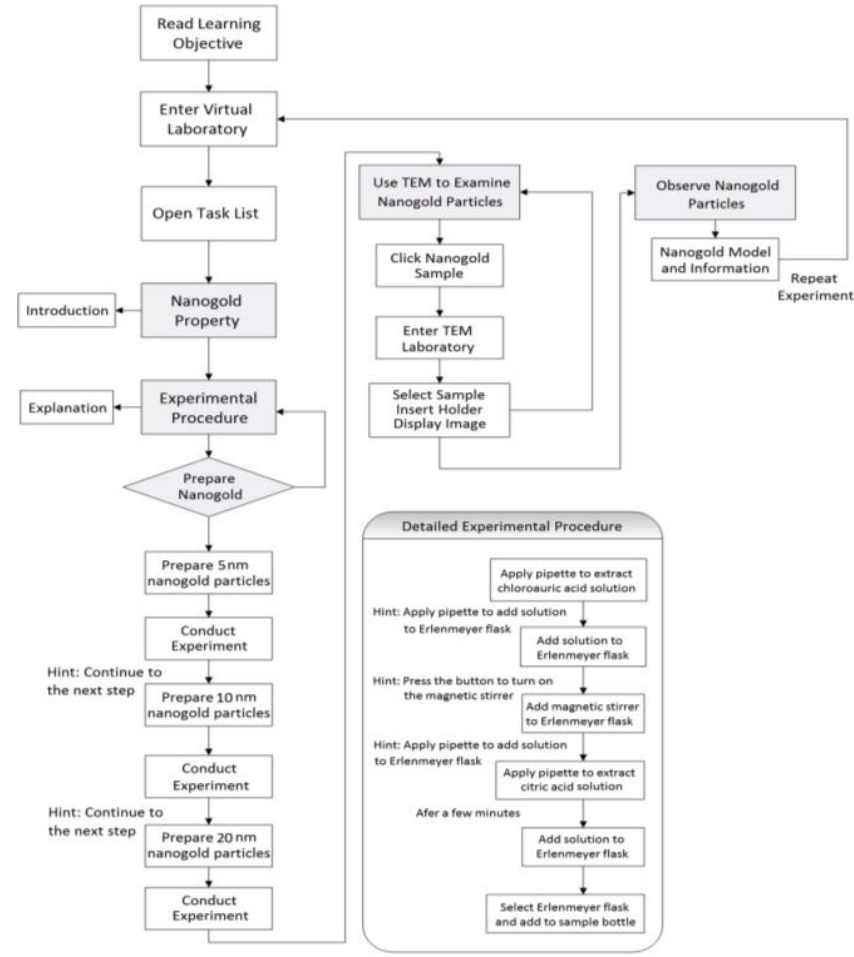

Fig. 1. The operational procedure for conducting the virtual experiment.
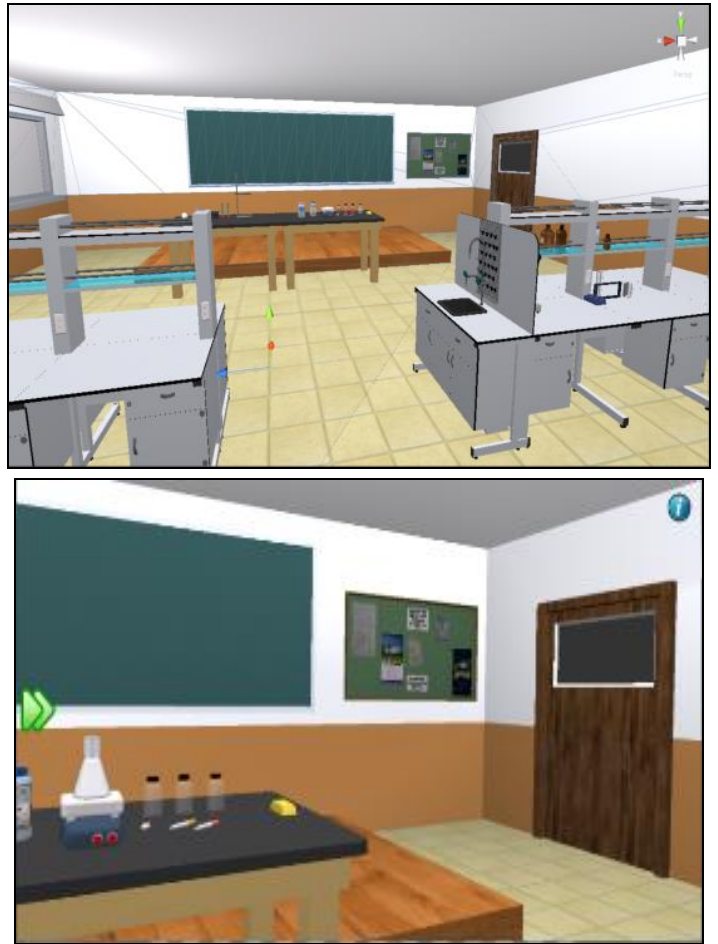

Fig. 2. The virtual laboratory and its instruments.

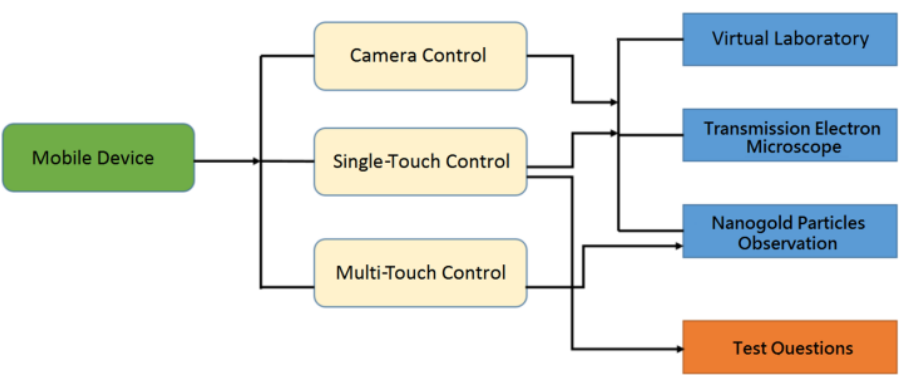

Fig. 3. The system architecture of virtual laboratory.

The operational interface of virtual laboratory is designed by combining the virtual reality technology and the touch screen functions on mobile devices to satisfy the requirement of situated learning. This study used 3ds Max and Unity 3D to create the virtual scene of the virtual laboratory and designed the interactive user interface respectively. The users can follow the verbal instructions to select the virtual instruments for conducting the experiment. After opening the task list, the users can see the major steps to complete the experiment. They can proceed by following the experimental procedure shown on the task list, and the system will record the learning status for each step. Finally, the users can take the online test after completing the experiment to evaluate their learning achievements. The operational procedure for the virtual experiment is shown in Fig. 1.

\subsection{System Architecture}


The development of virtual laboratory is composed of two major parts. The first part is to design the 3D models of instruments in the virtual laboratory, and the second part is to set up the interactive interface for the user to manipulate these objects. First, the virtual scene of the laboratory and its instruments were designed by 3ds Max (Fig. 2). After that, the 3D models were exported to Unity 3D to set up the interactive user interface according to the script defining the operational procedure of the virtual experiment. The design of user interface contains three modules: the camera control, single-touch control, and multi-touch control. This study used the C\# programming language and the internal API functions to develop the transmission electron microscope for the observation of nanogold particles, and online test modules in the virtual laboratory (Fig. 3).

\subsection{Virtual Experiment}

The virtual laboratory is designed for execution on mobile devices as well as personal computers (Fig. 4). After entering the system, the verbal instruction will inform the users to open the task list by clicking the triangular button on the left side to see the steps for completing the experiment (Fig. 5). The users can read the learning objective (Fig. 6) of the virtual experiment by clicking the Information button in the upper right corner. They can also study the property of nanogold by selecting the first task (Fig. 7).

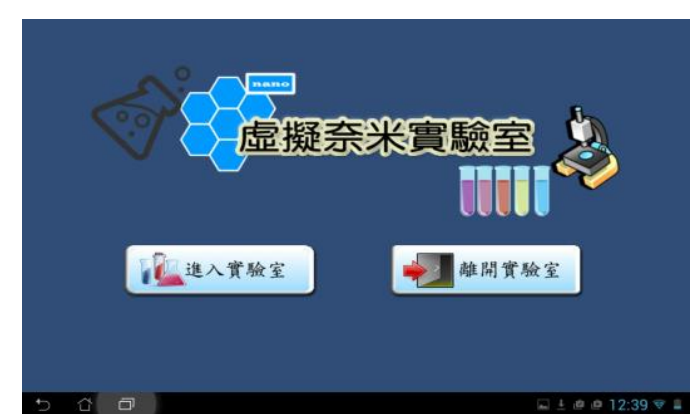

Fig. 4. Enter the virtual laboratory.

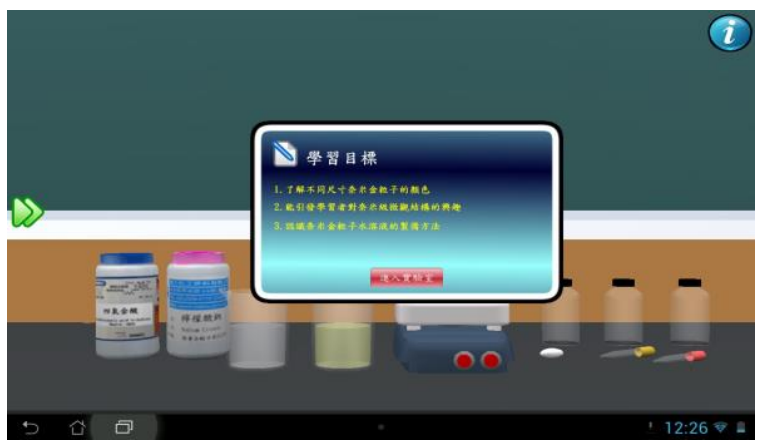

Fig. 6. Read the learning objective.

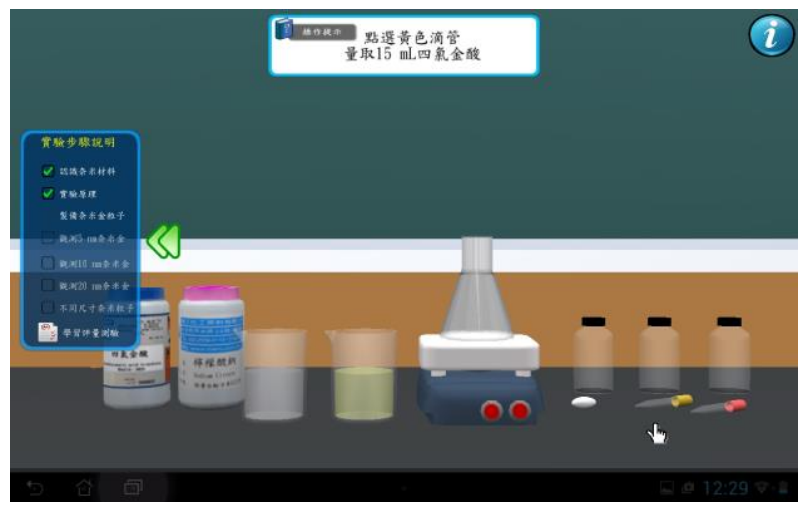

Fig. 8. Select the yellow pipette.

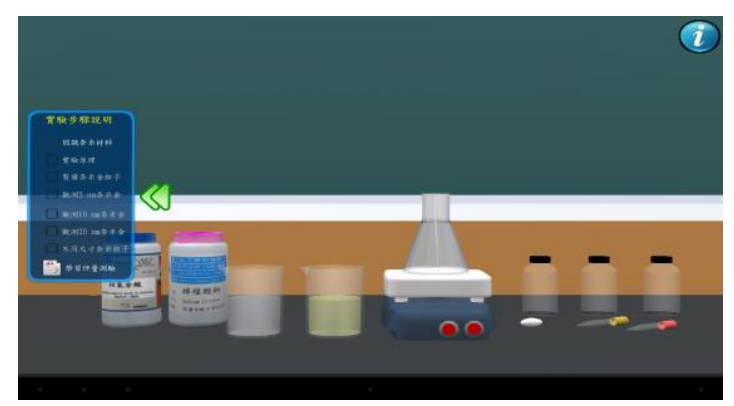

Fig. 5. Open the task list on the left.

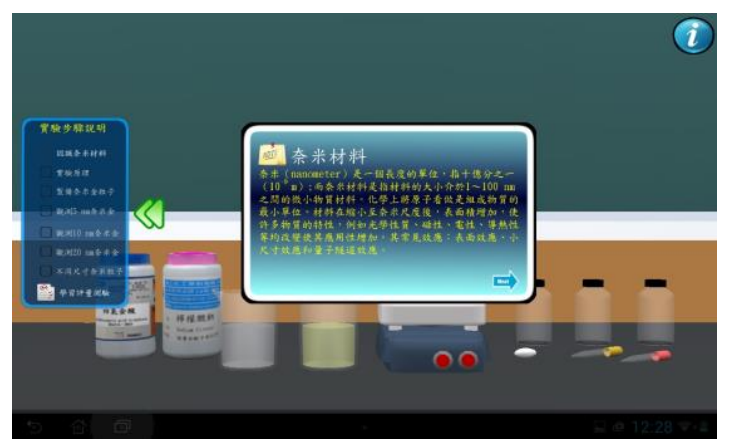

Fig. 7. Study the property of nanogold.

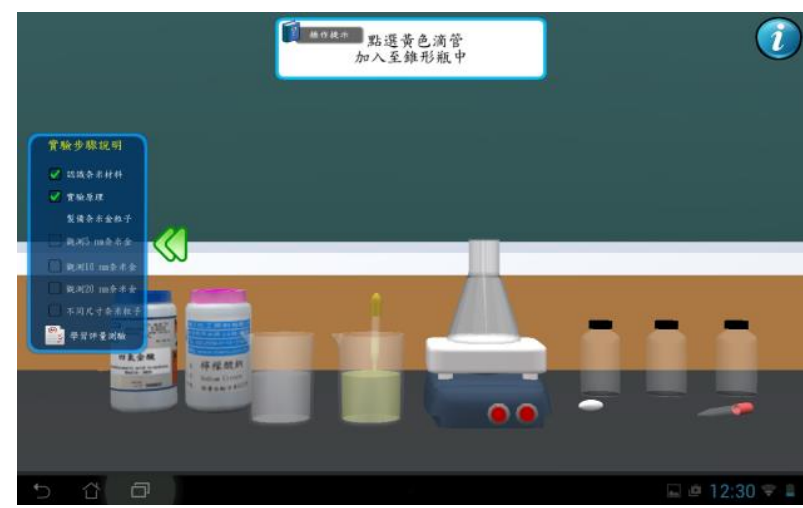

Fig. 9. Take $15 \mathrm{ml}$ of chloroauric acid. 
To begin with the synthesis of nanogold, an online instruction will be shown on the screen to remind the users of selecting correct instruments for conducting the experiment. Following the instruction, the users can complete the preparation of nanogold samples step by step till the end of the experiment (Figs. 8-16). For users to understand the process of chemical reaction, the system provides them with a magnifying glass (symbolling the observation tool) to see the atomic world inside the Erlenmeyer flask after mixing the trisodium citrate with chloroauric acid solution. In this way, they can realize how nanogold particles are produced in the chemical reaction. Three types of nanogold samples, i.e., $5 \mathrm{~nm}, 10 \mathrm{~nm}$, and $20 \mathrm{~nm}$ in sizes are produced from the virtual experiment. By clicking the sample for testing, the users can proceed to the step where the nanogold particles can be observed using the virtual transmission electron microscope. The users can complete the observation by following the online instructions. After moving the sample to the holder and plugging the holder into the transmission electron microscope, the screen will display the 3D structures of nanogold particles by scanning the samples (Figs. 17-19). When the scanned images of nanogold particles have been obtained, the users can compare their 3D structures and properties (Figs. 20-23).

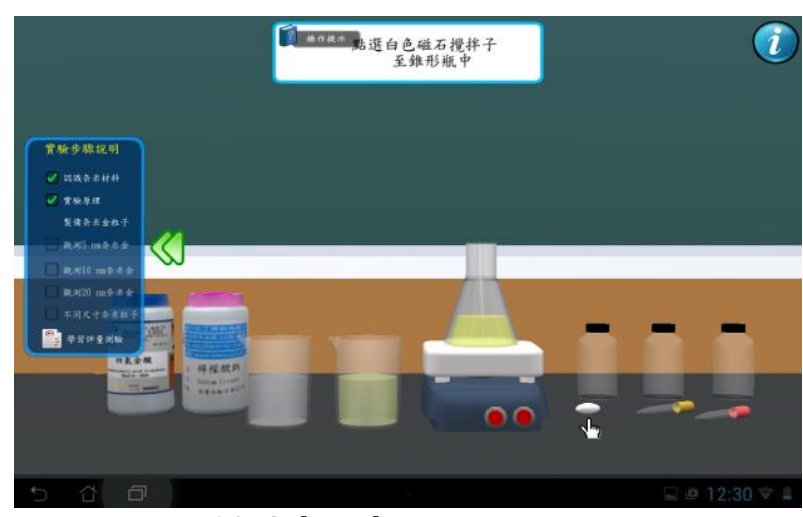

Fig. 10. Select the magnetic stirrer.

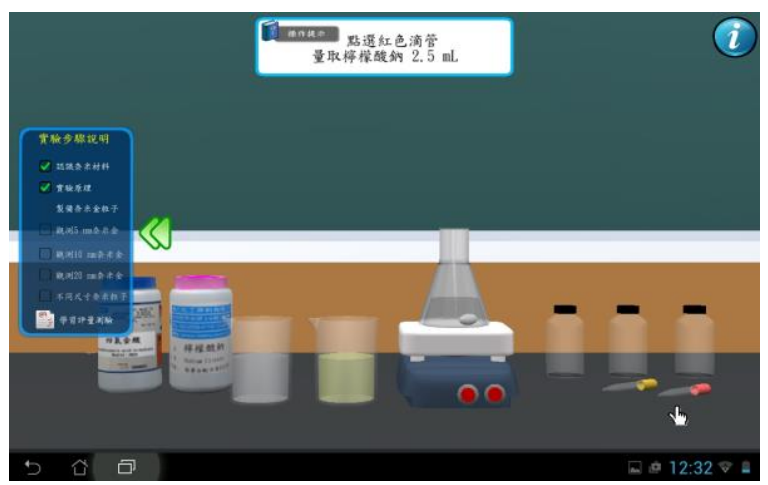

Fig. 12. Select the red pipette.

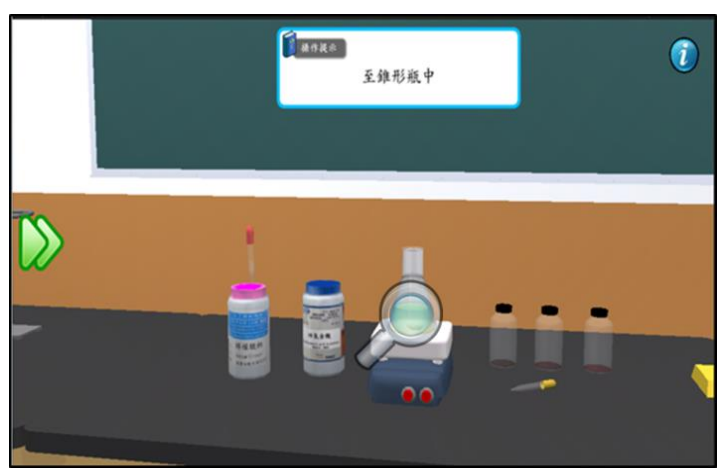

Fig. 14. Observe the chemical reaction.

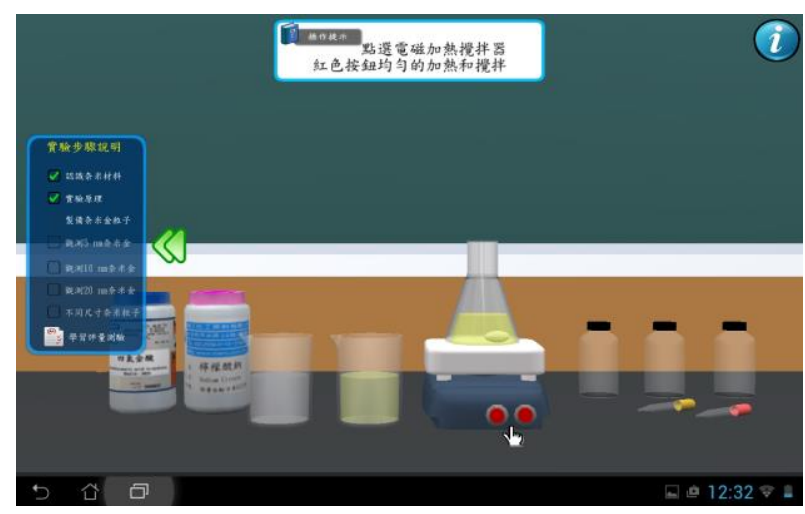

Fig. 11. Turn on the hot plate and magnetic stirrer.

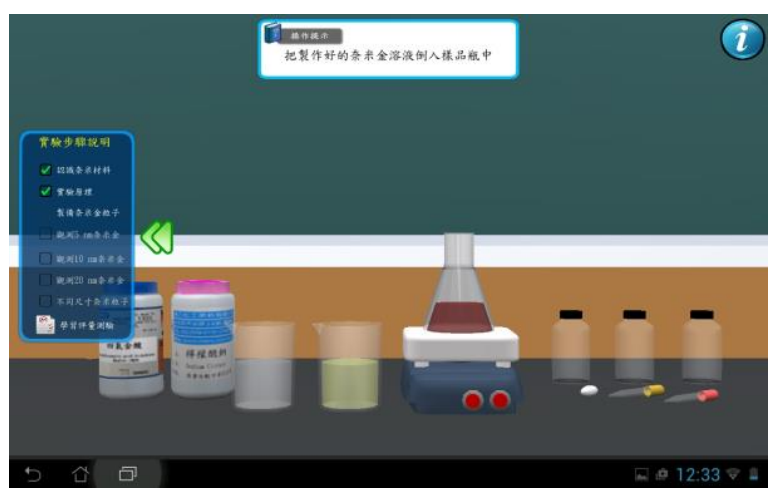

Fig. 13. Synthesize the nanogold solution.

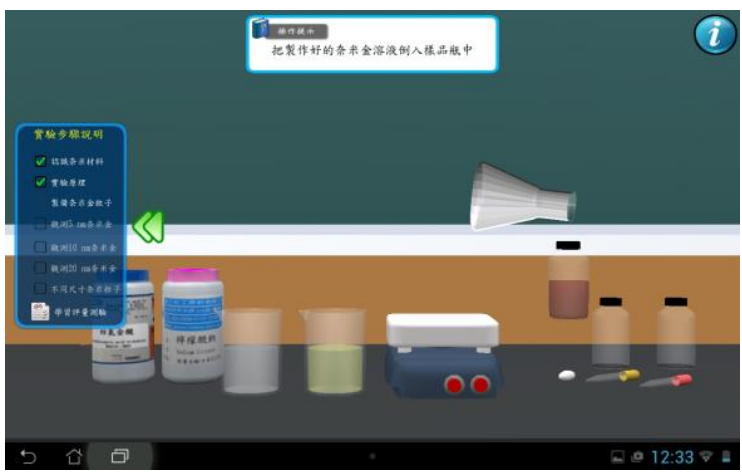

Fig. 15. Put nanogold solution into a bottle. 


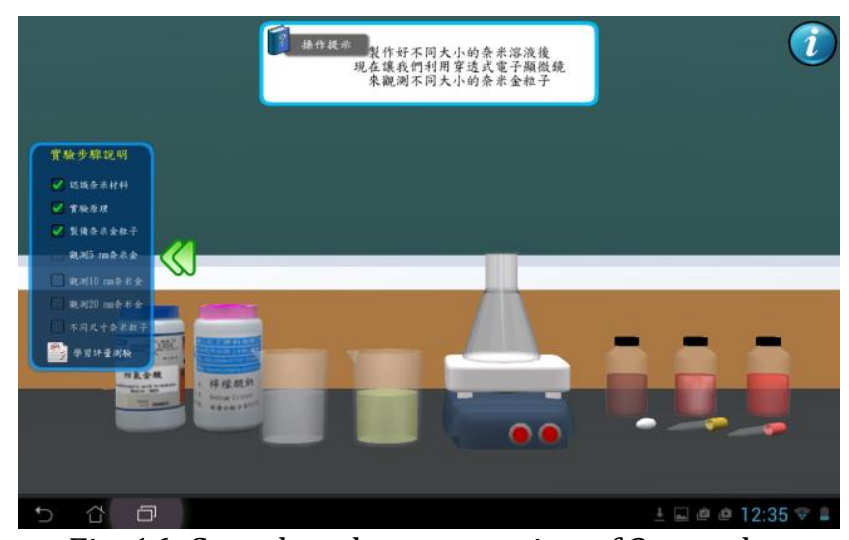

Fig. 16. Complete the preparation of 3 samples.

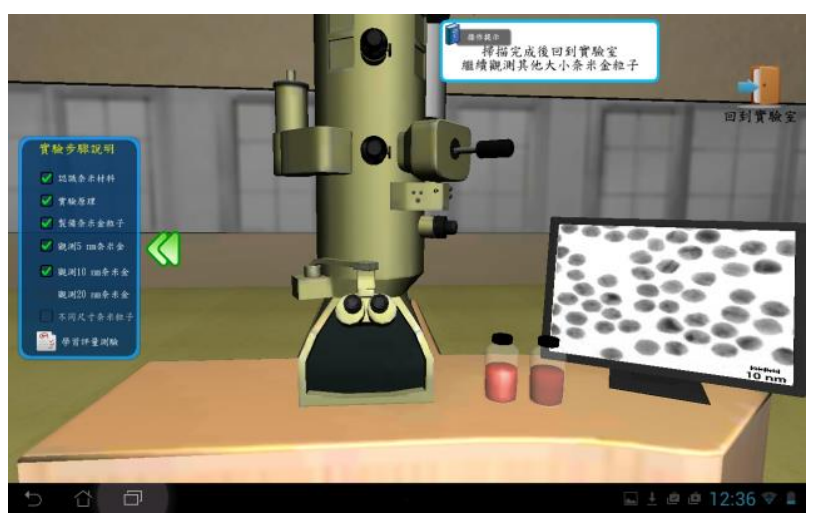

Fig. 18. Scan the nanogold sample (10nm).

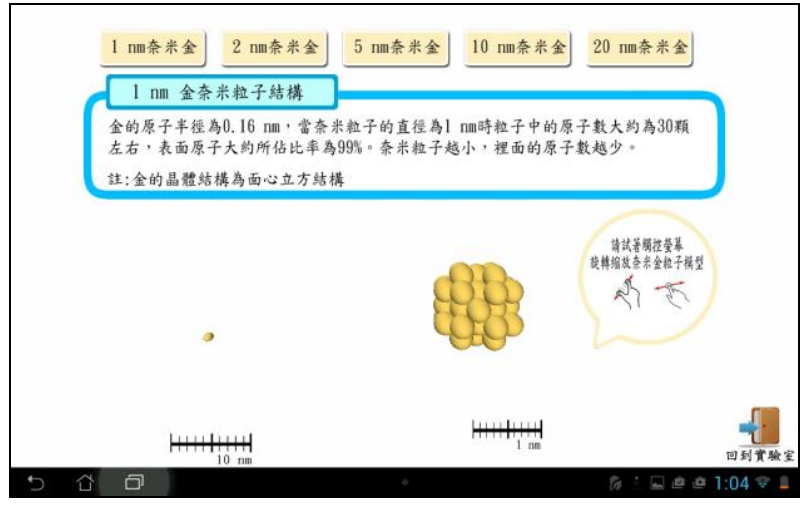

Fig. 20. The 3D structure of $1 \mathrm{~nm}$ nanogold.

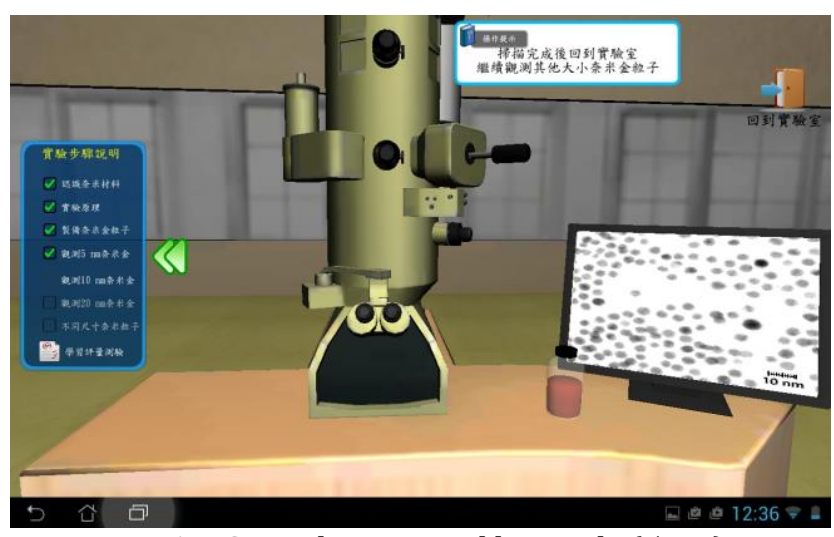

Fig. 17. Scan the nanogold sample $(5 \mathrm{~nm})$.

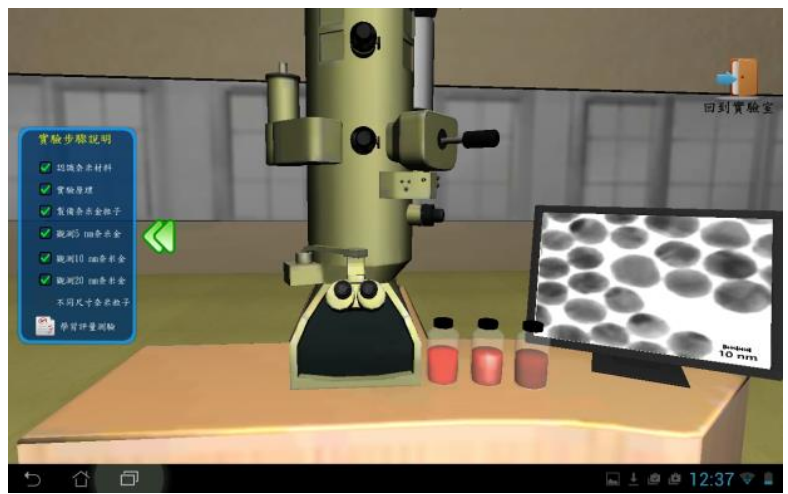

Fig. 19. Scan the nanogold sample (20nm).

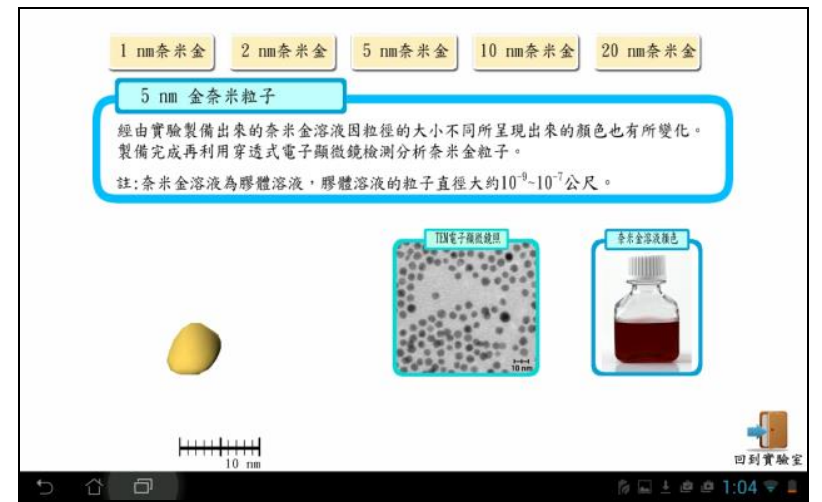

Fig. 21. The 3D structure of 5nm nanogold.

\subsection{Online Test}

If the users have completed the synthesizing and analyzing steps on the task list (Fig. 24), the system will request them to take the online test to evaluate their learning achievements. By clicking the Test button, the system will display the test questions for users to answer (Fig. 25). If the users select the correct answer, the system will display "You are right." (Fig. 26) and move to the next question; if they select the wrong answer, the system will display "You are wrong." and show the correct answer to explain the reason (Fig. 27). Finally, the users can click the Exit button in the upper right corner to return to the virtual laboratory.

\section{Teaching Experiment}

A teaching experiment has been conducted to evaluate the performance of virtual laboratory for students in learning the procedure of synthesizing and analyzing nanogold particles. A questionnaire survey has been conducted to investigate the attitudes of students after using the virtual laboratory. This study 
randomly selected 80 students from the department of applied science in a university in northern Taiwan as the experimental samples. The students were randomly divided into two groups, the control group (40 students, 21 males and 19 females) and the experimental group (40 students, 25 males and 15 females). After explaining the experimental procedure to both groups, the control group conducted the real experiment (Fig. 28) while the experimental group conducted the virtual experiment (Fig. 29) respectively.

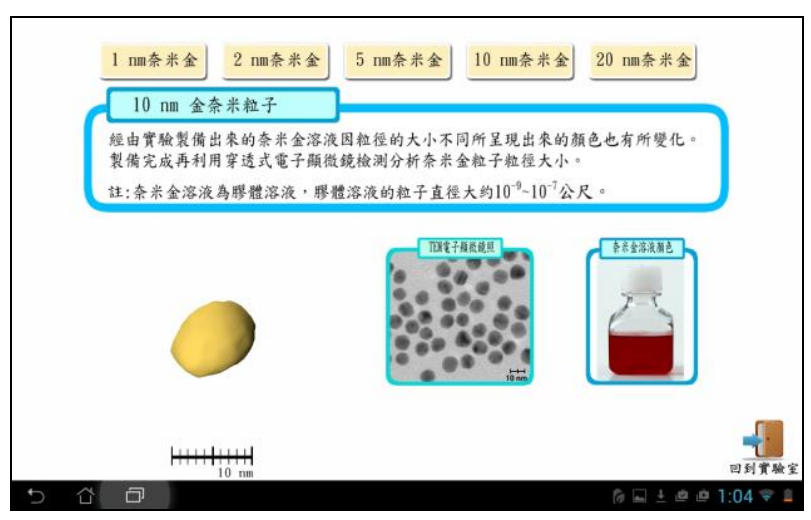

Fig. 22. The 3D structure of 10nm nanogold.

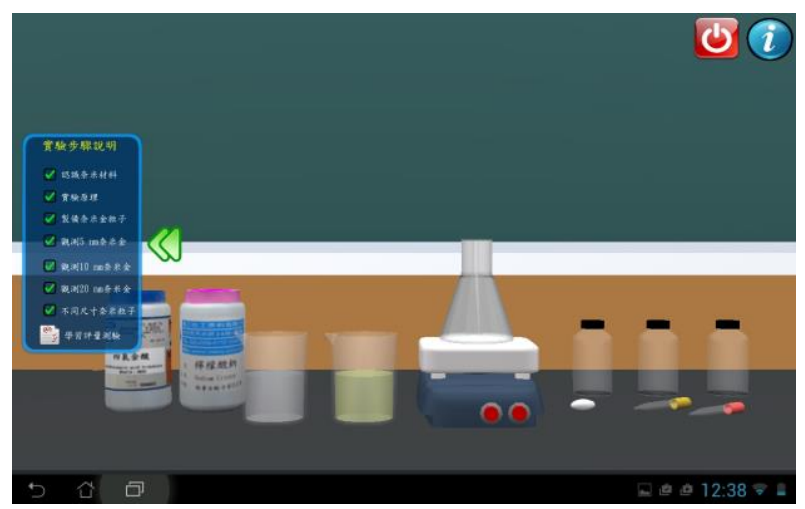

Fig. 24. Complete the virtual experiment.

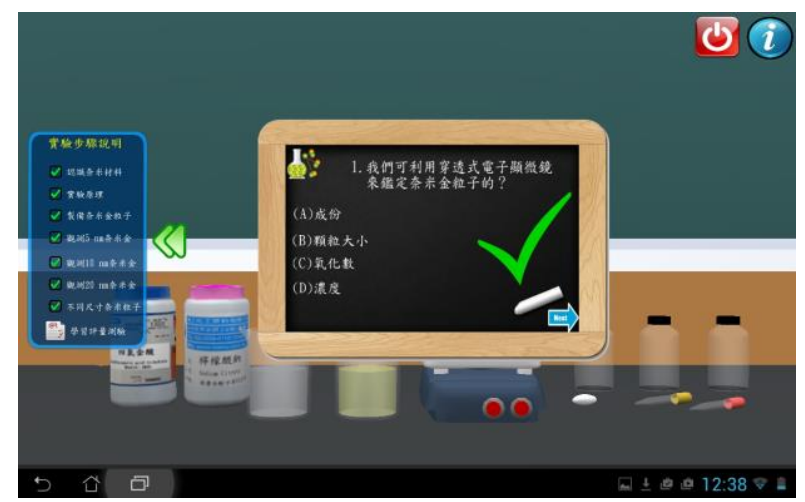

Fig. 26. The answer is correct.

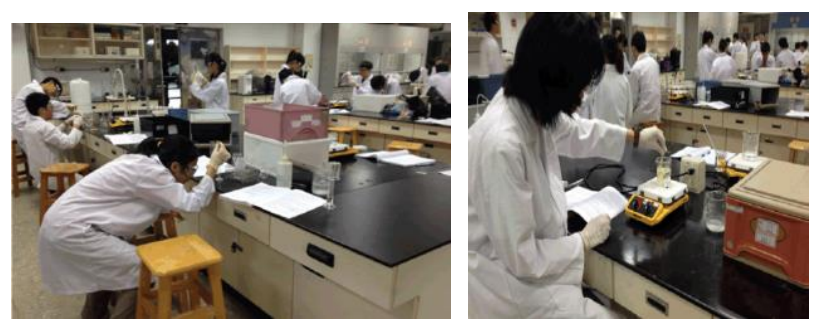

Fig. 28. The control group conducted the real experiment.

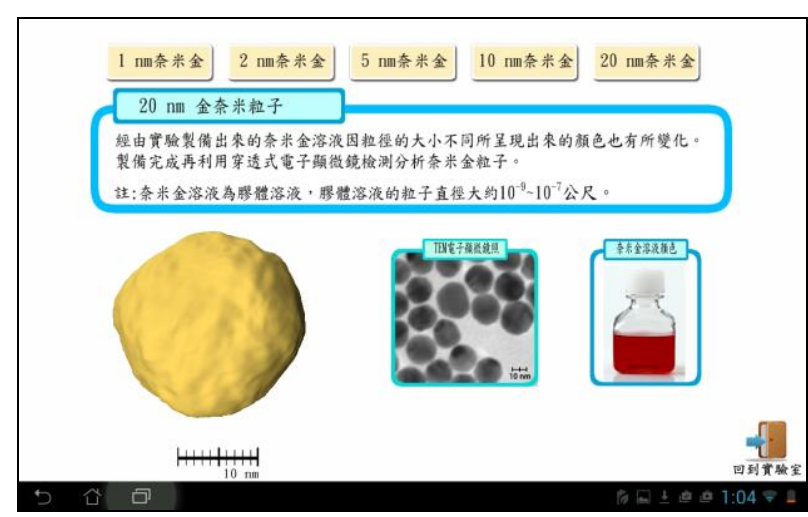

Fig. 23. The 3D structure of $20 \mathrm{~nm}$ nanogold.

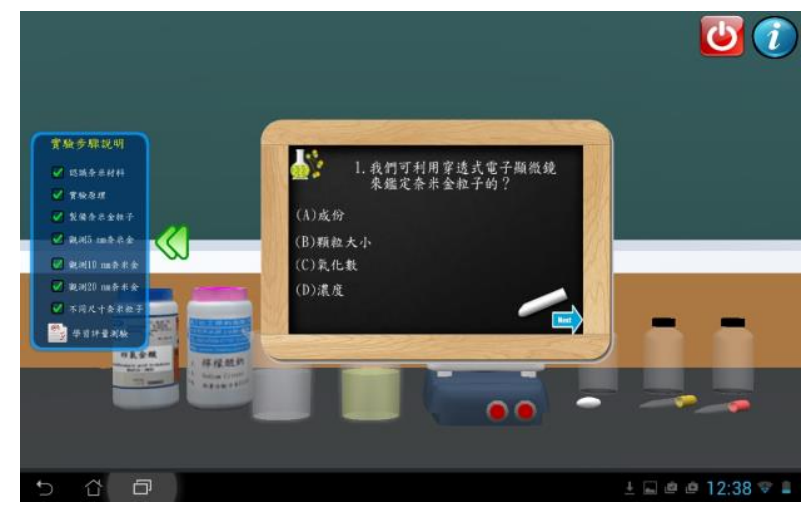

Fig. 25. Answer the test question.

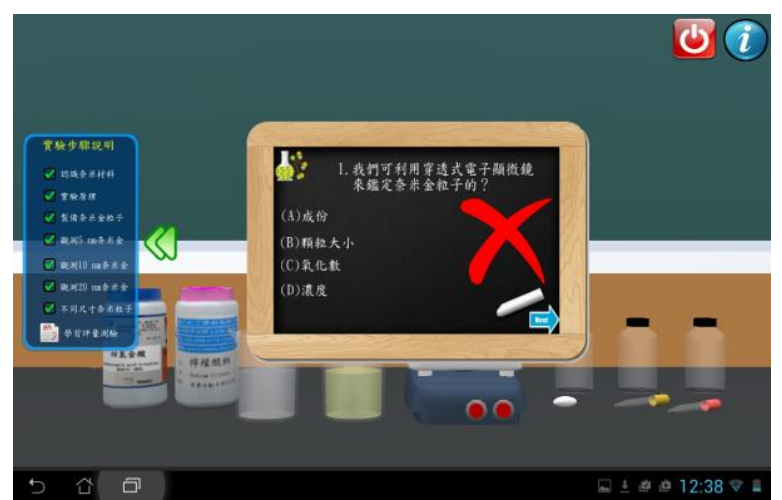

Fig. 27. The answer is wrong.
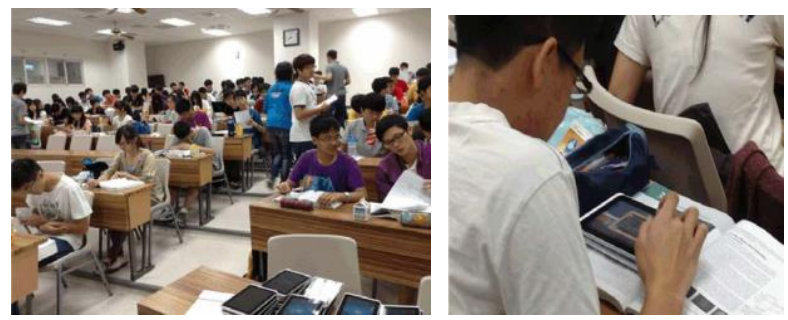

Fig. 29. The experimental group conducted the virtual experiment. 
This study adopted a nonequivalent pretest-posttest design to investigate if there is a significant difference in learning effectiveness between the two groups. Before teaching activities, all students took a pretest on their background knowledge about nanogold. After conducting the chemical experiment, they took the posttest and the experimental group also filled out a questionnaire. The variables in the teaching experiment are shown in Table 1, and the flowchart of the teaching experiment is shown in Fig. 30.

Table 1. The Variables of Teaching Experiment

\begin{tabular}{ccc}
\hline \hline \multirow{2}{*}{ Independent Variables } & Control Group (40 university students) & Experiment using the real laboratory \\
\cline { 2 - 3 } & Experimental Group (40 university students) & Experiment using the virtual laboratory \\
\hline Covariance & Achievement test (pretest) \\
\hline Dependent Variable & Achievement test (posttest) \\
\hline Control Variable & Teacher, teaching time, learning contents \\
\hline \hline
\end{tabular}

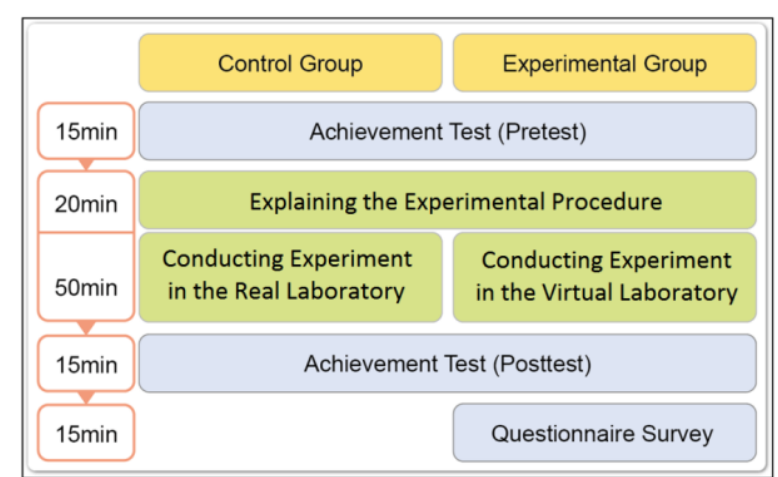

Fig. 30. The flowchart of teaching experiment.

\subsection{Research Tools}

The research tools used in this study include the virtual laboratory, the real laboratory, the instructional PowerPoint to explain the experimental procedure, achievement test (pretest and posttest), and questionnaire survey, which are described briefly in the following:

- Virtual Laboratory

The virtual laboratory is an app for execution on mobile devices and its contents are designed based on the real experiment for preparing and analyzing nanogold particles. The system was installed on tablet PCs and used by students in the experimental group.

- Real Laboratory

The real laboratory is a chemistry laboratory in the Department of Applied Science at a university in northern Taiwan, and it was used for conducting the experiment of synthesizing and analyzing nanogold particles.

\section{- Achievement Test}

The achievement test used in this study contains 20 test items which were designed based on the research goal, teaching objectives and learning contents. They were revised according to the comments of experts and science teachers.

\section{- Questionnaire Survey}

The questionnaire survey used in this study is a system satisfaction survey according to the user's experiences after conducting the virtual experiment, and it was designed based on the discussion of experts and science teachers. The questionnaire contained 17 questions (5 questions in learning contents, 5 questions in interface design, and 7 questions in system operational experience). This study adopted Likert's 5-point scale (scoring: 5=strongly agree; 4=agree; $3=$ neutral; $2=$ disagree; $1=$ =strongly disagree) [23] to measure the attitudes of students after using the system. The statistical software SPSS was used to perform a reliability analysis and the value of Cronbach's alpha was calculated as 0.833 , showing the results 
has met the standard of high reliability.

\subsection{Learning Achievement Analysis}

In the teaching experiment, the control group used the real laboratory and the experimental group used the virtual laboratory to conduct the chemical experiment. An analysis of covariance (ANCOVA) has been conducted to investigate if there is a significant difference in learning effectiveness between the two groups. The statistical software SPSS is used to analyze the test results (Table 2). For the control group, the average scores of pretest and posttest are 60.75 and 73.38 respectively. For the experimental group, the average scores of pretest and posttest are 66.00 and 87.63 respectively. The progress made by the experimental group (21.63) is higher than the progress made by the control group (12.63).

Table 2. Descriptive Statistics of Achievement Test

\begin{tabular}{cccccc}
\hline \hline & Achievement Test & Degree of Freedom & Mean & Standard Error & Standard Deviation \\
\hline \multirow{2}{*}{ Control Group } & Pretest & 39 & 60.75 & 1.45 & 8.96 \\
\cline { 2 - 6 } & Posttest & 39 & 73.38 & 1.08 & 6.83 \\
\hline \multirow{2}{*}{ Experimental Group } & Pretest & 39 & 66.00 & 1.71 & 10.81 \\
\cline { 2 - 6 } & Posttest & 39 & 87.63 & 1.12 & 7.07 \\
\hline \hline
\end{tabular}

This study used a Levene's test to compare the pretest results to see if any significant difference about the background knowledge of nanogold experiment existed between the two groups, and the results show that $F=2.376$ and $P=0.127>0.05$ (significant standard), indicating there is no significant difference and therefore their background knowledge is about the same.

To investigate if students made significant progress after learning, this study used a paired sample T-test to perform a statistical analysis on the pretest and posttest for both groups (Table 3). For the control group, the T value is 13.33 and the significance $P$ is less than 0.001 , indicating the real laboratory is effective to enhance the learning achievement of control group. For the experimental group students, the $T$ value is 16.23 and the significance $P$ is less than 0.001 , indicating the virtual experiment can also enhance their learning achievements.

Table 3. Results of Paired Sample T-test

\begin{tabular}{cccccc}
\hline \hline & Mean of Progress & Standard Deviation & $\mathrm{T}$ & Degree of Freedom & Significance (P) \\
\hline Control Group & 12.63 & 5.99 & 13.33 & 39 & $<.001^{*}$ \\
Experimental Group & 21.63 & 8.43 & 16.23 & 39 & $<.001^{*}$ \\
\hline \hline
\end{tabular}

Before performing the ANCOVA to compare the learning effectiveness by two groups, the assumptions for the homogeneity of variance and within-group regression coefficients have to be satisfied. The statistical results for the above analyses are described in the following:

- Homogeneity of Variance

This study used a Levene's test to verify if there is homogeneity of variance between the two groups. According to the test results, the value of $F=2.167$ and the significance $\mathrm{P}=0.145<0.05$, indicating the variance has not achieved the standard of significance and thus the assumption for the homogeneity of variance is satisfied.

- Homogeneity of Within-group Regression Coefficients

Before performing the ANCOVA, the test for homogeneity of within-group regression coefficients must be carried out for the two groups. The results show that $F=1.743$ and $P=0.191>0.05$, also without achieving the standard of significance. Thus, the dependent variables and covariance are not influenced by the independent variable and the assumption is satisfied, so the ANCOVA can be continued.

- ANCOVA Results

This study used an ANCOVA to compare the difference in learning achievements between the control 
group and the experimental group. After excluding the effect of covariance and dependent variables, the impact of independent variable (the teaching tools) is obtained as $F=96.788$ and $P=0.001<0.05$. A significant difference exists between the control group and experimental group, and the learning achievement of the latter is higher than that of the former. In other words, the virtual laboratory is more effective than the real laboratory for enhancing the learning achievement of students.

\subsection{Questionnaire Results}

This study carried out a statistical analysis on questionnaire results by the experimental group as the system satisfaction survey. The percentage of student number and the average score in each selected item are computed for all questions to understand the attitudes of students after using the virtual laboratory (Table 4).

Table 4. Questionnaire Results of Experimental Group

\begin{tabular}{|c|c|c|c|c|c|c|c|}
\hline 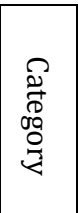 & Evaluation Item & 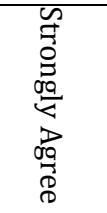 & $\begin{array}{l}\vec{D} \\
\text { 贾 } \\
0\end{array}$ & 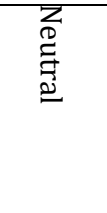 & 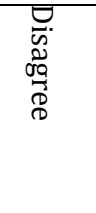 & 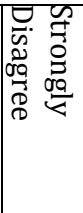 & 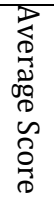 \\
\hline \multirow{5}{*}{ 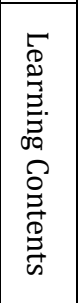 } & 1. I can understand most textual descriptions in the virtual laboratory. & $55 \%$ & $45 \%$ & $0 \%$ & $0 \%$ & $0 \%$ & 4.6 \\
\hline & $\begin{array}{l}\text { 2. I can understand the procedure and principle of preparing nanogold } \\
\text { particles after using the virtual laboratory. }\end{array}$ & $40 \%$ & $50 \%$ & $10 \%$ & $0 \%$ & $0 \%$ & 4.3 \\
\hline & 3. The virtual laboratory provides adequate prompts. & $35 \%$ & $52.5 \%$ & $12.5 \%$ & $0 \%$ & $0 \%$ & 4.2 \\
\hline & $\begin{array}{l}\text { 4. The virtual laboratory provides the relative knowledge about } \\
\text { nanotechnology. }\end{array}$ & $67.5 \%$ & $30 \%$ & $2.5 \%$ & $0 \%$ & $0 \%$ & 4.3 \\
\hline & $\begin{array}{l}\text { 5. The virtual laboratory is educational and it can help learning } \\
\text { knowledge related to nanotechnology. }\end{array}$ & $42.5 \%$ & $52.5 \%$ & $5 \%$ & $0 \%$ & $0 \%$ & 4.4 \\
\hline \multirow{5}{*}{ 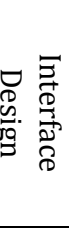 } & 6. It is easy to operate the system. & $32.5 \%$ & $50 \%$ & $17.5 \%$ & $0 \%$ & $0 \%$ & 4.2 \\
\hline & 7. The text in the virtual laboratory is clear. & $27.5 \%$ & $47.5 \%$ & $22.5 \%$ & $2.5 \%$ & $0 \%$ & 4.0 \\
\hline & $\begin{array}{ll}\text { 8. The experimental instruments in the virtual laboratory look very } \\
\text { realistic. }\end{array}$ & $22.5 \%$ & $45 \%$ & $32.5 \%$ & $0 \%$ & $0 \%$ & 3.9 \\
\hline & 9. The colors and design style are consistent. & $32.5 \%$ & $55 \%$ & $12.5 \%$ & $0 \%$ & $0 \%$ & 4.2 \\
\hline & 10. The operational speed of the system is proper. & $15 \%$ & $32.5 \%$ & $47.5 \%$ & $5 \%$ & $0 \%$ & 3.6 \\
\hline \multirow{7}{*}{ 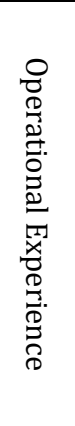 } & $\begin{array}{l}\text { 11. Using the virtual laboratory makes me understand the procedure } \\
\text { for preparing nanogold particles. }\end{array}$ & $42.5 \%$ & $55 \%$ & $2.5 \%$ & $0 \%$ & $0 \%$ & 4.4 \\
\hline & $\begin{array}{l}\text { 12. Using the virtual laboratory helps me understand the principle of } \\
\text { synthesizing nanogold particles. }\end{array}$ & $42.5 \%$ & $57.5 \%$ & $0 \%$ & $0 \%$ & $0 \%$ & 4.5 \\
\hline & 13. Using the virtual laboratory increases my interest in nanoscale. & $25 \%$ & $35 \%$ & $40 \%$ & $0 \%$ & $0 \%$ & 3.9 \\
\hline & $\begin{array}{l}\text { 14. Using the virtual laboratory makes me understand the related } \\
\text { knowledge about nanotechnology }\end{array}$ & $32.5 \%$ & $52.5 \%$ & $15 \%$ & $0 \%$ & $0 \%$ & 4.2 \\
\hline & $\begin{array}{l}\text { 15. In the virtual laboratory, I can see the 3D molecular model to } \\
\text { understand the structure of nanogold particles in different sizes. }\end{array}$ & $27.5 \%$ & $40 \%$ & $30 \%$ & $2.5 \%$ & $0 \%$ & 3.9 \\
\hline & 16. I have completed the mission and the test actively. & $30 \%$ & $47.4 \%$ & $22.5 \%$ & $0 \%$ & $0 \%$ & 4.1 \\
\hline & 17. I like to use to the virtual laboratory. & $27.5 \%$ & $45 \%$ & $27.5 \%$ & $0 \%$ & $0 \%$ & 4.0 \\
\hline
\end{tabular}

\section{- Learning Contents}

Most students thought they could understand the verbal and textual explanations in the virtual laboratory and learned the principle of synthesizing nanogold particles. They agreed that the virtual laboratory had adequate instructions and its contents provided them with useful knowledge for conducting the experiment. The statistical results reveal that the scores in this category are between 4.2 and 4.6, and the overall average score is 4.36 .

\section{- Interface Design}

Most students considered the operation of virtual laboratory is easy and the text in the virtual laboratory is clear. They thought the virtual laboratory and its instruments are realistic and the colors as well as the design style are consistent. The score for the operational speed is only 3.6 due to the fact that computers need more computation time in rendering the 3D virtual scenes and processing the interaction with users. 
The statistical results reveal that the scores in this category are between 3.6 and 4.2 , and the overall average score is 4 .

- Operating Experiences

Most students felt that they could understand the procedure and principle of synthesizing nanogold particles after conducting the virtual experiment. They had actively completed the experiment and test questions, and became more interested in learning nanotechnology. The completion of all tasks gave them a great sense of achievement, so they would like to use the virtual laboratory as a teaching tool in the future. The statistical results reveal that the scores in this category are between 3.9 and 4.5 , and the overall average score is 4.15 .

\section{Conclusion}

In this study, a virtual laboratory for synthesizing and analyzing nanogold particles has been developed for students to conduct a virtual experiment to become familiar with the procedure of synthesizing nanogold and understand the change in property due to its size. In the future, the augmented reality (AR) technology can also be applied to provide the user interface for motion-sensing operation by using the functions of three-axial accelerometer and electronic compass. In that case, the users can immerse into the learning situation by physical operation to enhance their learning interest and motivation.

The virtual laboratory is designed by combining the virtual reality technology and situated learning theory with the knowledge of chemical experiment for synthesizing and analyzing nanogold. The system can be executed on mobile devices as well as personal computers, and it has been applied in university chemistry courses to increase students' knowledge of nanotechnology through the operation of virtual experiments. They can become familiar with the experimental procedure and principle through interactive operation following the step-by-step instructions. The samples of nanogold particles can be examined using the virtual transmission electron microscope to enhance understanding about the property of nanogold particles.

A teaching experiment has been performed to investigate students' learning achievements and attitudes after using the virtual laboratory. The experimental results reveal that both the virtual and real experiments could effectively enhance their learning achievements. However, the learning effectiveness of the virtual laboratory is significantly higher than that of the real experiment. The questionnaire results have shown that most students were satisfied with the learning contents, interface design, and system operation. In addition, most students held positive attitudes towards the virtual laboratory and they considered it effective in improving their learning motivation and interest. They thought the system could help them understand the procedure and principle of synthesizing nanogold particles and acquire relevant knowledge through the analysis of their structure using the virtual transmission emission microscope.

\section{Acknowledgment}

The authors would like to thank for the financial support of Ministry of Science and Technology (MOST), Taiwan under the contract number 104-2514-S-134-003.

\section{References}

[1] Feynman, R. P. (1960). There's plenty of room at the bottom. Engineering and Science, 23(5), 22-36.

[2] National Science and Technology Museum. (2008). A New World in Nanotechnology. Retrieved July 19, 2004, from http://nano.nstm.gov.tw/

[3] Ministry of Education. (2008). General Supplementary Teaching Material: Introduction to Nanotechnology, K12 Educational Development Center in Central and Northern Taiwan.

[4] Drexler, K. E. (1992). Nanosystems: Molecular Machinery, Manufacturing, and Computation. New York: 
John Wiley \& Sons.

[5] Bird, P. (2006). U.S. National Nanotechnology Initiative. Retrieved November 17, 2015, from http://web2.clarkson.edu/projects/nanobird/3.4.php

[6] Ghosh, D. \& Chattopadhyay, N. (2013). Equilibrium and dynamic effects on ligand binding to bio macromolecules and biomimetic model systems. International Reviews of Physical Chemistry, 32, 435-466.

[7] Tsai, Y. C., Shieu, F. S., Lu, F. H., \& Wu, C. M. (2007). Atomic Force Microscope Practical Training Materials. Taipei: Wu-Nan (In Chinese).

[8] Neogy, S., Savalia, R. T., Tewari, R., Srivastava, D., \& Dey, G. K. (2006). Transmission electron microscopy of nanomaterials. Indian Journal of Pure \& Applied Physics, 44(2), 119-124.

[9] Rugar, D., Mamin, H. J., \& Guenther, P. (1990). Magnetic force microscopy: general principles and application to longitudinal recording media. Journal of Applied Physics, 68(3), 1169-1183.

[10] Vasir, J. K., Reddy, M. K., \& Labhasetwar, V. D. (2005) Nanosystems in drug targeting: Opportunities and challenges. Current Nanoscience, 1, 47-64.

[11] Turkevich, J., Stevenson, P. C., \& Hillier, J. (1953). The formation of colloidal gold. Journal of Physical Chemistry, 57, 670-673.

[12] Frens, G. (1973). Preparation of gold dispersions of varying size: Controlled nucleation for the regulation of the particle size in monodisperse gold suspensions. Nature: Physical Science, 241, 20-22.

[13] Heim, M. (1993). The Metaphysics of Virtual Reality, New York: Oxford University Press.

[14] Burdea, G. (1993). Virtual reality systems and applications. Proceedings of the Electro'93 International Conference, New Jersy, USA, 1993.

[15] Earnshaw, R. A., Vince, J. A., \& Jones, H. (1995). Virtual Reality Applications, San Diego: Academic Press.

[16] Bell, J. T., \& Fogler, H. S. (1996). Vicher: A virtual reality based educational module for chemical reaction engineering. Computer Applications in Engineering Education, 4(4), 285-296.

[17] Tarng, W., Lee, B. S., Lin, C. M., Lee, C. Y. \& Liou, H. H. (2012). Design of a physical game for learning the nanoconcepts of the butterfly effect. International Journal of Research and Reviews in Computer Science, 3(5), 1886-1893.

[18] Brown, J. S., Collins, A., \& Duguid, P. (1989). Situated cognition and the culture of learning. Educational Researcher, 18(1), 32-42.

[19] Kolb, D. (1984). Experiential Learning: Experience as the Source of Learning and Development, Englewood Cliffs, New Jersey: Prentice Hall.

[20] Dewey, J. (1963). Experience and Education, New York: Collier Books.

[21] Wang, R. K. (2009). Applications of situated learning in elementary school English teaching. Taipei County Education, 69, 87-91.

[22] Collins, A. (1989). Cognitive Apprenticeship and Instructional Technology, Center for the Study of Reading, University of Illinois at Urbana. Champaign.

[23] Likert, R. (1932). A Technique for the Measurement of Attitudes. New York: Archives of Psychology.

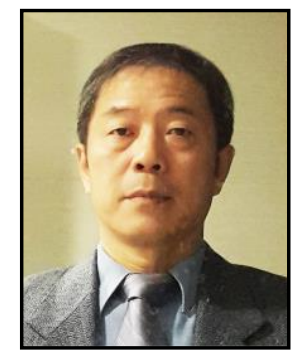

Wernhuar Tarng is a professor at the Graduate Institute of Human Resource and e-Learning Technology, National Hsinchu University of Education, Taiwan. He was the chairman of the Graduate Institute of Computer Science from 2008 to 2012 . He graduated from the Department of Control Engineering at National Chiao Tung University, Hsinchu, Taiwan in 1984, and received his M.S. and Ph.D. degrees in Electrical and Computer Engineering from State University of New York at Buffalo, the U.S.A. in 1987 and 1992, respectively. He was a visiting scholar at Oxford University, the 
United Kingdom in 2003. His research interests include computer network, image processing, real-time systems, virtual reality, and e-Learning technology.
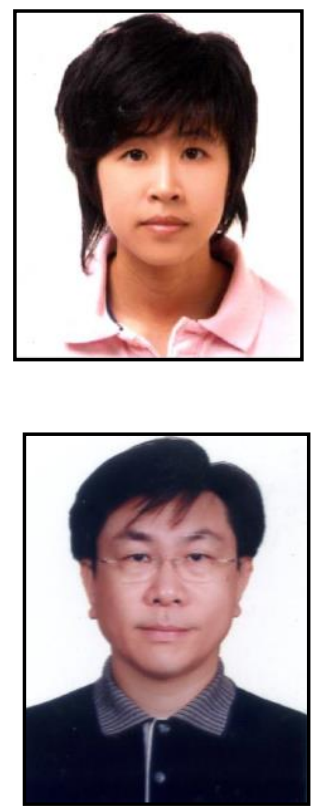

Chia Chun Hsie received her B.S. degree from the Department of Information Management at Shih Hsin University, Taipei, Taiwan in 2008. She received her M.S. degree from the Graduate Institute of Human Resource and e-Learning Technology at National Hsinchu University of Education, Taiwan in 2015. She is now an assistant researcher at Industrial Technology Research Institute (ITRI), Hsinchu, Taiwan. Her research interests include information management, virtual reality and e-Learning technology.

Chih Ming Lin is a professor at the Department of Applied Science and the director of Library and Information Services Center, National Hsinchu University of Education, Taiwan. He received his B.S. degree from the Department of Physics, National Changhua University of Education, Taiwan in 1984, M.S. degree from the Department of Physics, Tamkang University, Taipei, Taiwan in 1989, and Ph.D. degree from the Department of Electrophysics, National Chiao Tung University, Hsinchu, Taiwan in 1997. In the last ten years, he has conducted research at National Synchrotron Light Source (NSLS), Advanced Light Source (ALS) in the U.S.A. and SPring8 in Japan. His research interests include investigating physical properties under high-pressure highly correlated phase transition of nanocrystalline and thermal electric materials.

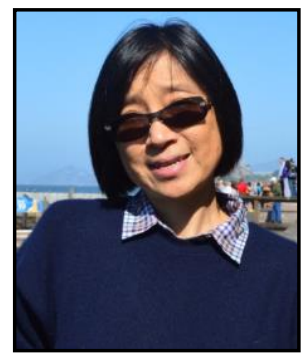

Chi-Young Lee is a professor at the Department of Materials Science and Engineering, National Tsing Hua University, Hsinchu, Taiwan. She is the director of Interdisciplinary Program of Tsing Hua from 2015. She graduated from the Department of Chemistry at National Tsing Hua University in 1977 and received her M.S. and Ph. D. degrees from the Institute of Chemistry, National Tsing Hua University in 1979 and 1986, respectively. Her research interests include synthesis of high performance materials by using green chemical methods and development of teaching materials for science education. 